Pacific Journal of Mathematics

BERNSTEIN-LIKE POLYNOMIAL APPROXIMATION IN 


\title{
BERNSTEIN-LIKE POLYNOMIAL APPROXIMATION IN HIGHER DIMENSIONS
}

\author{
LESTER E. DUBINS
}

Let $C(K)$ be the Banach space of continuous, real-valued functions defined on a compact, Hausorff space, $K$, let $\mathscr{P}=\mathscr{P}(K)$ be the positive linear forms, $P$, defined on $C(K)$, for which $P f \leq \sup f(k)(k \in K)$, $f \in C(K)$, and endow $\mathscr{P}(K)$ with the weak-star topology in which it is, of course, compact. (As is well known, $\mathscr{P}$ can be identified with the set of countably additive probability measures defined on the Baire subsets of $K$.) Let $P^{\infty}$ be the power probability on $K^{\infty}$, the product of denumerable number of copies of $K$. Then, for each $f \in C\left(K^{\infty}\right)$, the integral of $f$ with respect to $P^{\infty}$ is plainly continuous in $P$. As Theorem 2 below states, there are no ohter continuous real-valued functions of $P$. The proof of this assertion requires a generalization of Bernstein's version of the celebrated polynomial approximation theorem of Weierstrass, which generalization is provided by Theorem 1 .

The two theorems are numbered in their logical order but stated in the order of simplicity of formulation.

THEOREM 2. For every $g \in C(\mathscr{P}(K))$, there is an $f \in C\left(K^{\infty}\right)$ such that, for all $P \in \mathscr{P}(K)$,

$$
\int f d P^{\infty}=g(P) .
$$

It is convenient to reformulate Theorem 2 in terms of an operator $T$ mapping $C\left(K^{\infty}\right)$ into $C(\mathscr{P}(K))$ defined, thus.

$$
(T f) P=\int f d P^{\infty}, \quad f \in C\left(K^{\infty}\right), P \in \mathscr{P}(K) .
$$

Reformulated, Theorem 2, states that the operator $T$ is surjective, that is, onto $C(\mathscr{P})$. A short digression explains the origin of this theorem.

Suppose $Q$ is a probabilistic mixture of power probabilities,

$$
Q=\int P^{\infty} \mu(d P)
$$

for some probability $\mu$ on $\mathscr{P}$, or, more fully,

$$
Q f=\int(T f)(P) \mu(d P), \quad f \in C(K) .
$$


As a theorem of Hewitt and Savage [2] asserts, each $Q$ is representable in at most one way as a mixture of power probabilities. Plainly, for the uniqueness of $\mu$, it is sufficient that every $g \in C(\mathscr{P})$ be in the uniform closure of functions of the form $T f$ for $f \in C(K)$. It was this observation which led me to investigate whether even the stronger assertion of Theorem 2 might be true, which explains the genesis of this paper.

To Benjamin Weiss ${ }^{1}$ I am quite indebted. For it was he who, in a private communication to me, proved Theorem 2 when $K$ is a two-point set. Moreover, his argument carries over to arbitrary compact sets, $K$, once Bernstein's polynomial approximation theorem is generalized, as in Theorem 1 below.

Recall that Bernstein has shown that, for each continuous function, $g$, defined on the closed unit interval, the polynomials $g_{n}$ converge uniformly to $g$ where

$$
g_{n}(p)=\sum_{j=1}^{n}\left(\begin{array}{l}
n \\
j
\end{array}\right) g\left(\frac{j}{n}\right) p^{\jmath}(1-p)^{n-j} .
$$

To state the generalization of Bernstein's theorem, introduce the usual notation, $\delta_{k}$, for the probability supported by the singleton $\{k\}$, that is, $\delta_{k} \in \mathscr{P}(K)$ is the evaluation map: $\phi \rightarrow \phi(k)$, for $\phi \in C(K)$. Letting $\omega=(k(1), k(2), \ldots)$ be a generic point of $K^{\infty}$, introduce $D_{n}(\omega)$, its empirical distribution of order $n$,

$$
D_{n}(k(1), k(2), \ldots)=\frac{1}{n} \sum_{i=1}^{n} \delta_{k(l)} .
$$

Of course, $D_{n}: K^{\infty} \rightarrow \mathcal{P}$ is continuous. Therefore, its composition with a $g \in C(\mathscr{P}), g \circ D_{n}$, also to be designated by $S_{n} g$, is a continuous, real-valued mapping with domain $K^{\infty}$ which has an expectation under any probability on $K^{\infty}$, in particular, under any power probability $P^{\infty}$. Recapitulating, $S_{n}: C(\mathcal{P}) \rightarrow C\left(K^{\infty}\right)$ and $T S_{n}: C(\mathcal{P}) \rightarrow C(\mathscr{P})$ where $T$ is defined as in (2). In fuller detail,

$$
\left(T S_{n} g\right) P=\int S_{n} g d P^{\infty}=\int g \circ D_{n} d P^{\infty},
$$

for $g \in C(\mathscr{P}), P \in \mathscr{P}$.

THEOREM 1. TS $S_{n}$ converges in the strong operator topology to the identity on $C(\mathscr{P})$, that is, for each $g \in C(\mathcal{P})$, (7) converges to $g(P)$ uniformly in $P$.

\footnotetext{
${ }^{1}$ Rather than being a coauthor, Weiss invited me to use the ideas and the argument contained in his communication.
} 
Of course, if $K$ is a two-point set $\{\mathrm{a}, \mathrm{b}\}, \mathscr{P}$ is identified with the closed unit interval via the correspondence $P \Leftrightarrow p$ if, and only if, $P(b)=p$, and, in this case, $T S_{n} g$ is the Bernstein polynomial $g_{n}$ of (5). If $K$ is a finite set, $\mathcal{P}$ is a simplex and one obtains from Theorem 1 similar polynomial approximation to $g$. For instance, if $K$ is a three-point set, $\Delta$ is the triangle of all triplets $(p, q, r)$ of nonnegative numbers whose sum is $1, g \in C(\Delta)$.

$$
g_{n}(p, q, r)=\sum\left(\begin{array}{c}
n \\
i, j, k
\end{array}\right) g\left(\frac{i}{n}, \frac{j}{n}, \frac{k}{n}\right) p^{l} q^{j} r^{k}
$$

where the sum is over all triplets of nonnegative integers whose sum is $n$, and where the familiar multinomial notation is being used; and $g_{n}$ converges to $g$ uniformly on $\Delta$.

It is an immediate corollary of Theorem 1 that the range of $T$ is dense in $C(\mathcal{P})$ which, of course, yields another proof of the Hewitt and Savage [2] result which asserts the uniqueness of the representation of an exchangeable probability as a mixture of power probabilities.

2. Quadratic functions. If $Y$ is a topological linear space, later to be specialized to be the dual of $C(K)$ in the usual weak-star topology, a finite linear combination of functions, each of which is either a constant, a continuous linear functional defined on $Y$, or the square of such a linear function, is a finite-rank quadratic function. The restriction of a quadratic function to a subset of $Y$ is called a quadratic function on that subset. Throughout this note, $Y$ is endowed with the weakest topology which permits each element $x$ of a linear space $X$ of linear functionals on $Y$ to be continuous.

LEMMA 1. Let $y \in V \subset Y$, where $V$ is a neighborhood of $y$, and let $b$ and $c$ be real numbers. Then there is a finite-rank quadratic function $q: Y \rightarrow \mathbf{R}$ such that $q(y)=b, q \geq b$ everywhere, and $q \geq c$ on the complement of $V$.

Proof of Lemma 1. Since the space of finite-rank quadratic functions is invariant under translations of $Y$, as well as under positive affine transformations of $\mathbf{R}$, it suffices to treat the case where $y$ is the origin of $Y, b=0$, and $c=1$. Since $Y$ has the weak topology induced by $X$, there exist $x_{i} \in X, 1 \leq i \leq n$, such that $\left|\left(x_{l}, z\right)\right|<1$ for all $i$ implies $z \in V$. Plainly, for $q(z)=\Sigma\left(x_{i}, z\right)^{2}, q(0)=0, q \geq 0$ everywhere, and $q \geq 1$ on the complement of $V$. 
LEMMA 2. Let $g$ be a bounded function defined on a subset $L$ of $Y$ which, at a point $y \in L$, is continuous (or even semicontinuous). Then, for every $\varepsilon>0$, there is a finite-rank quadratic function $q$ on $L$ which majorizes $g$ and which, at $y$, is $g(y)+\varepsilon$.

Proof. Let $V$ be a neighborhood of $y$ such that, on $V, g$ is nowhere greater than $g(y)+\varepsilon$. The preceding lemma applies with $b=g(y)+\varepsilon$ and $c$ equal to any upper bound for $g$.

Let $J$ be the set of finite-rank quadratic functions on $L$.

Lemma 3. Let $g \in C(L)$ where $L$ is a compact subset of $Y$. Then

$$
g(y)=\inf \{q(y) \mid q \geq g, q \in J\},
$$

and

$$
g(y)=\sup \{q(y) \mid q \leq g, q \in J\} .
$$

Proof. From Lemma 2, (1) is immediate, and (2) follows by applying (1) to $-g$.

Proposition 1. Let $L$ be a compact subset of $Y$, and let $T_{1}, T_{2}, \ldots$ be a sequence of order-preserving (not necessarily linear) mappings of $C(L)$ into itself such that $T_{n} q$ converges to $q$ whenever $q$ is a finite-rank quadratic function. Then $T_{n}$ converges in the strong operator topology to the identity operator, that is, for each $g \in C(L), T_{n} g$ converges to $g$ uniformly on $L$.

Proof. Let $g \in C(L)$. Then as Lemma 3 asserts, $g$ satisfies (1) and (2). And, as shown by Bauer [1, Proposition 1] which in turn was inspired by results of Korovkin [3], for every $g$ which satisfies (1) and (2), $T_{n} g \rightarrow g$ uniformly on $L$. This completes the proof, but for the convenience of the reader, here is a sketch of the argument that $T_{n} g \rightarrow g$. First, use (1) and (2), and the compactness of $L$ to verify that, for every $\varepsilon>0$, there exist $q_{i} \in J, 1 \leq i \leq m$, each of which majorizes $g$, but whose infimum is majorized by $g+\varepsilon$. By hypothesis, $\exists N$ such that $T_{n} q_{i}<q_{l}+\varepsilon$ for $n \geq N$ and all $i$. Since $T_{n}$ is order-preserving, one obtains

$$
T_{n} g<\inf _{i} T_{n} q_{i}<\inf _{i} q_{i}+\varepsilon<g+2 \varepsilon .
$$

A similar calculation shows that, for $n \geq N^{\prime}, T_{n} g$ exceeds $g-2 \varepsilon$. 
3. Proof of Theorem 1. Fix $\phi \in C(K)$ and let

$$
v_{\phi} P=\int \phi^{2} d P-\left(\int \phi d P\right)^{2}
$$

which is often called the variance of $\phi$ with respect to $P$, and let

$$
q_{\phi} P=\left(\int \phi d P\right)^{2}
$$

LEMMA 1. $T S_{n} q_{\phi}=q_{\phi}+\frac{1}{n} V_{\phi}$.

The following straightforward calculation comprises the proof of Lemma 1.

$$
\begin{aligned}
\left(T S_{n} q_{\phi}\right) P & =\int q_{\phi} \circ D_{n} d P^{\infty} \\
= & \int\left(\frac{1}{n} \sum \phi\left(k_{i}\right)\right)^{2} d P^{\infty} \\
= & \frac{1}{n^{2}}\left\{\int \sum_{i \neq j} \phi\left(k_{i}\right) \phi\left(k_{J}\right)+\sum \phi^{2}\left(k_{i}\right)\right\} d P^{\infty} \\
= & \int \phi\left(k_{1}\right) \phi\left(k_{2}\right) d P^{2}+\frac{1}{n}\left\{\int \phi^{2} d P-\int \phi\left(k_{1}\right) \phi\left(k_{2}\right) d P^{2}\right\} \\
= & \left(\int \phi d P\right)^{2}+\frac{1}{n}\left(\int \phi^{2} d P-\left(\int \phi d P\right)^{2}\right)
\end{aligned}
$$

(Incidentally, with the exception of the last equality, all equalities obtain if $P^{\infty}$ were any exchangeable probability or, more generally, any second-order exchangeable probability.)

Let $Y$ be the dual of $C(K)$ and endow $Y$ with the weak-star topology. As is well known, functions of the form $P \rightarrow \int \phi d P$ for some $\phi \in C(K)$ are the only continuous linear functionals on $Y$. As is verified without difficulty, if $g$ is such a function, or is constant, then $T S_{n} g=g$ for all $n$. This, together with Lemma 1, implies that $T S_{n} g \rightarrow g$ uniformly on $\mathcal{P}$ whenever $g$ is a finite-rank quadratic function. Now Proposition 1 of the preceding section applies.

\section{Proof of Theorem 2.}

LEMMA 1. Let $T$ be a bounded linear transformation of a Banach space $X$ into a normed linear space $Z$. Then, for $T$ to be surjective (onto $Z$ ), it suffices that there exist a mapping $S$, not necessarily linear or continuous, of 
$Z$ into $X$ and positive numbers $\alpha$ and $\beta, \alpha<1$, such that, for all $g \in Z$,

$$
\|g-T S g\| \leq \alpha\|g\|
$$

and

$$
\|S g\| \leq \beta\|g\| \text {. }
$$

Proof of Lemma 1. Fix a $g \in Z$ and define a mapping $U=U_{g}$ of $X$ into $X$, thus.

$$
U x=x+S(g-T x) .
$$

Since $U^{n+1} x=U^{n} x+S\left(g-T U^{n} x\right)$ and, since $T$ is linear,

$$
\begin{aligned}
\left\|g-T U^{n+1} x\right\| & =\left\|g-T U^{n} x-T S\left(g-T U^{n} x\right)\right\| \\
& \leq \alpha\left\|g-T U^{n} x\right\| \leq \alpha^{n+1}\|g-T x\|,
\end{aligned}
$$

where (1) and an induction have been used. Consequently, for all $x$,

$$
\lim T U^{n} x=g .
$$

Next, use (3) and (2) to obtain

$$
\|U x-x\|=\|S(g-T x)\| \leq \beta\|g-T x\| .
$$

Now use (6) and (4) to get

$$
\left\|U^{n+1} x-U^{n} x\right\| \leq \beta\left\|g-T U^{n} x\right\| \leq \beta \alpha^{n}\|g-T x\| .
$$

Therefore, $\lim U^{n} x$ exists and

$$
T\left(\lim U^{n} x\right)=\lim T U^{n} x=g
$$

where the continuity of $T$ and (5) have been used.

Define a mapping $S$ of $C(\mathscr{P})$ into $C\left(K^{\infty}\right)$ by letting $S(g)$ be $S_{n} g=$ $g\left(D_{n}\right)$ when $n=n(g)$ is minimal with the property

$$
\left\|g-T S_{n} g\right\| \leq \frac{1}{2}\|g\| \text {. }
$$

That $S$ is well defined is implied by Theorem 1. Plainly, for each $\omega \in K^{\infty},\left(S_{n} g\right)(\omega)=g\left(D_{n}(\omega)\right)$, so the range of $S_{n} g$ is a subset of the range of $g$ which implies that

$$
\left\|S_{n} g\right\| \leq\|g\| \text {. }
$$

Since (9) and (10) plainly hold with $S_{n}$ replaced by $S$, the condition of Lemma 1 obtains with $X=C\left(K^{\infty}\right), Z=C(\mathscr{P}), \alpha=\frac{1}{2}$ and $\beta=1$. 
Further acknowledgements. In addition to Benjamin Weiss, I am indebted to David Aldous, Paul Chernoff, Michael Fochler, Avram Neyman and Gideon Schwartz for various helpful conversations and suggestions.

\section{REFERENCES}

[1] Heinze Bauer, Approximation and abstract boundaries, Amer. Math. Monthly, 85, No. $8,(1978), 632-647$.

[2] E. Hewitt and L. J. Savage, Symmetric measures on Cartesian products, Trans. Amer. Math. Soc., 80 (1955), 470-501.

[3] P. P. Korovkin, Linear Operators and Approximation Theory, Hindustan Publishing Corporation, Delhi, India, (1960).

Received November 19, 1981. Research supported by NSF Grant No. MCS-80-02535.

UNIVERSITY OF CALIFORNIA

BERKELEY, CA 94720 



\title{
PACIFIC JOURNAL OF MATHEMATICS EDITORS
}

\author{
Donald BabBitT (Managing Editor) \\ University of California \\ Los Angeles, CA 90024 \\ Hugo RossI \\ University of Utah \\ Salt Lake City, UT 84112 \\ C. C. Moore and Arthur Ogus \\ University of California \\ Berkeley, CA 94720
}

J. DugundiI

Department of Mathematics

University of Southern California

Los Angeles, CA 90089-1113

R. FinN and H. SAMELSON

Stanford University

Stanford, CA 94305

\section{ASSOCIATE EDITORS}
R. ARENS
E. F. BECKENBACH
B. H. NeUmanN
F. WOLF
K. YosHida
(1906-1982)

\section{SUPPORTING INSTITUTIONS}

\author{
UNIVERSITY OF ARIZONA \\ UNIVERSITY OF BRITISH COLUMBIA \\ CALIFORNIA INSTITUTE OF TECHNOLOGY \\ UNIVERSITY OF CALIFORNIA \\ MONTANA STATE UNIVERSITY \\ UNIVERSITY OF NEVADA, RENO \\ NEW MEXICO STATE UNIVERSITY \\ OREGON STATE UNIVERSITY
}

\author{
UNIVERSITY OF OREGON \\ UNIVERSITY OF SOUTHERN CALIFORNIA \\ STANFORD UNIVERSITY \\ UNIVERSITY OF HAWAII \\ UNIVERSITY OF TOKYO \\ UNIVERSITY OF UTAH \\ WASHINGTON STATE UNIVERSITY \\ UNIVERSITY OF WASHINGTON
}

The Supporting Institutions listed above contribute to the cost of publication of this Journal, but they are not owners or publishers and have no responsibility for its content or policies.

Mathematical papers intended for publication in the Pacific Journal of Mathematics should be in typed form or offset-reproduced (not dittoed), double spaced with large margins. Please do not use built up fractions in the text of the manuscript. However, you may use them in the displayed equations. Underline Greek letters in red, German in green, and script in blue. The first paragraph must be capable of being used separately as a synopsis of the entire paper. In particular it should contain no bibliographic references. Please propose a heading for the odd numbered pages of less than 35 characters. Manuscripts, in triplicate, may be sent to any one of the editors. Please classify according to the scheme of Math. Reviews, Index to Vol. 39. Supply name and address of author to whom proofs should be sent. All other communications should be addressed to the managing editor, or Elaine Barth, University of California, Los Angeles, California 90024.

There are page-charges associated with articles appearing in the Pacific Journal of Mathematics. These charges are expected to be paid by the author's University, Government Agency or Company. If the author or authors do not have access to such Institutional support these charges are waived. Single authors will receive 50 free reprints; joint authors will receive a total of 100 free reprints. Additional copies may be obtained at cost in multiples of 50 .

The Pacific Journal of Mathematics is issued monthly as of January 1966. Regular subscription rate: $\$ 132.00$ a year (6 Vol., 12 issues). Special rate: $\$ 66.00$ a year to individual members of supporting institutions.

Subscriptions, orders for numbers issued in the last three calendar years, and changes of address should be sent to Pacific Journal of Mathematics, P.O. Box 969, Carmel Valley, CA 93924, U.S.A. Old back numbers obtainable from Kraus Periodicals Co., Route 100, Millwood, NY 10546.

The Pacific Journal of Mathematics ISSN $0030-8730$ is published monthly by the Pacific Journal of Mathematics at P.O. Box 969, Carmel Valley, CA 93924. Application to mail at Second-class postage rates is pending at Carmel Valley, California, and additional mailing offices. Postmaster: Send address changes to Pacific Journal of Mathematics, P. O. Box 969, Carmel Valley, CA 93924.

PUBLISHED BY PACIFIC JOURNAL OF MATHEMATICS, A NON-PROFIT CORPORATION

Copyright $\odot 1983$ by Pacific Journal of Mathematics 


\section{Pacific Journal of Mathematics}

Vol. 109, No. $2 \quad$ June, 1983

Tibor Bisztriczky, On the singularities of almost-simple plane curves . . . . 257

Peter B. Borwein, On Sylvester's problem and Haar spaces .............. 275

Emilio Bujalance, Cyclic groups of automorphisms of compact nonorientable Klein surfaces without boundary ............... 279

Robert Jay Daverman and John J. Walsh, Acyclic decompositions of

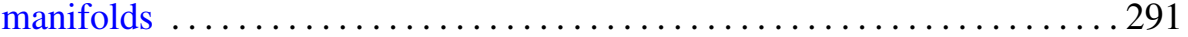

Lester Eli Dubins, Bernstein-like polynomial approximation in higher

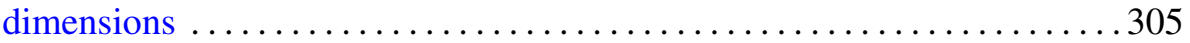

Allan L. Edelson and Jerry Dee Schuur, Nonoscillatory solutions of

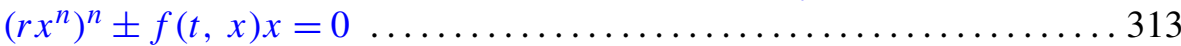

Akira Endô, On units of pure quartic number fields ................. 327

Hector O. Fattorini, A note on fractional derivatives of semigroups and

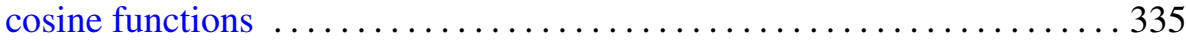

Ronald Fintushel and Peter Sie Pao, Circle actions on homotopy spheres with codimension 4 fixed point set ........................ 349

Stephen Michael Gagola, Jr., Characters vanishing on all but two

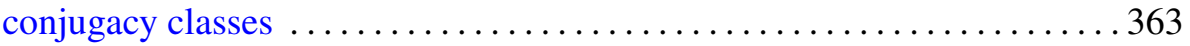

Saverio Giulini, Singular characters and their $L^{p}$ norms on classical Lie

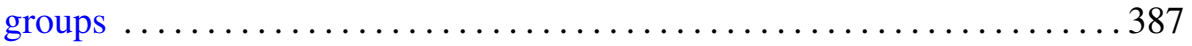

Willy Govaerts, Locally convex spaces of non-Archimedean valued continuous functions

Wu-Chung Hsiang and Bjørn Jahren, A remark on the isotopy classes of diffeomorphisms of lens spaces $\ldots . \ldots \ldots \ldots \ldots \ldots$

Hae Soo Oh, Compact connected Lie groups acting on simply connected 4-manifolds

Frank Okoh and Frank A. Zorzitto, Subsystems of the polynomial system

Knut Øyma, An interpolation theorem for $H_{E}^{\infty}$

Nikolaos S. Papageorgiou, Nonsmooth analysis on partially ordered vector spaces. II. Nonconvex case, Clarke's theory ........ 